\title{
O dilema dos métodos de custeio e das ferramentas de gerenciamento de custos: Uma
} proposta conceitual

\author{
The dilemma of cost methods and cost management tools: A conceptual proposal \\ EI dilema de los métodos de costos y las herramientas de gestión de costos: Una propuesta conceptual
}

Recebido: 23/07/2021 | Revisado: 27/07/2021 | Aceito: 28/07/2021 | Publicado: 29/07/2021

\author{
Marcelo Bernardino Araújo \\ ORCID: https://orcid.org/0000-0003-3211-6510 \\ Universidade Cruzeiro do Sul, Brasil \\ E-mail: marcelo.araujo@cruzeirodosul.edu.br \\ Everton Aristides Margueiro \\ ORCID: https://orcid.org/0000-0001-8118-7679 \\ Universidade Anhembi Morumbi, Brasil \\ E-mail: emargueiro@gmail.com \\ Marcos de Oliveira Morais \\ ORCID: https://orcid.org/0000-0002-5981-4725 \\ Universidade Estácio de Sá, Brasil \\ Universidade Santo Amaro, Brasil \\ E-mail: marcostecnologia2001@gmail.com
}

\begin{abstract}
Resumo
Importantes avanços da ciência contábil estiveram intimamente atrelados aos da sociedade e das empresas, especialmente após o século XIX, onde o aprimoramento das técnicas de apuração de custos se tornou evidentes e necessários. Desde então, notáveis contribuições emergiram e respondem pelo substrato dessa linha conceitual, até os dias atuais. No entanto não se constata na literatura nacional e internacional, estudos teóricos que apresentem uma separação objetiva entre os Métodos de Custeio e Ferramentas de Gestão. A constatação da falta de estudos que considerem as Ferramentas de Gerenciamento de Custos como elementos complementares aos Métodos de Custeios procuram-se responder às questões: Como os Métodos de Custeio e as Ferramentas de Gerenciamento são tratados nos estudos? Qual a tendência metodológica utilizada nos estudos que abordam a temática de custos? Qual a frequência com que os Métodos de Custeio e as Ferramentas de Gerenciamento são utilizados nos estudos? O artigo faz uma separação clássica dos conceitos teóricos dos Métodos de Custeio e das Ferramentas de Gerenciamento de Custos. As Ferramentas de Gerenciamento de Custos se distinguem dos Métodos de Custeio e, quando eleitas pelo pesquisador, podem ser usadas de maneira complementar aos Métodos de Custeio, suplantando o rigor das análises; e propiciando maior grau de segurança à apurações de custos, com vistas à tomada de decisão.
\end{abstract}

Palavras-chave: Sistemas de custos; Técnicas de custeio; Controle de gastos.

\begin{abstract}
Important advances in accounting science were closely linked to those of society and companies, especially after the 19 th century, when the improvement of cost calculation techniques became evident and necessary. Since then, notable contributions have emerged and account for the substrate of this conceptual line, until today. However, there is no evidence in the national and international literature, theoretical studies that present an objective separation between Costing Methods and Management Tools. The verification of the lack of studies that consider the Cost Management Tools as complementary elements to the Costing Methods seeks to answer the questions: How are the Costing Methods and the Management Tools treated in the studies? What is the methodological trend used in studies that address the theme costs? How often are Costing Methods and Management Tools used in studies? The article makes a classic separation of the theoretical concepts of Costing Methods and Cost Management Tools. The Cost Management Tools are different from the Costing Methods and, when chosen by the researcher, they can be used in a complementary way to the Costing Methods, supplanting the rigor of the analyses; and providing a greater degree of security in the calculation of costs, with a view to decision-making.
\end{abstract}

Keywords: Cost systems; Costing techniques; Control of expenses.

\section{Resumen}

Los importantes avances de la ciencia contable estuvieron estrechamente ligados a los de la sociedad y las empresas, especialmente a partir del siglo XIX, cuando la mejora de las técnicas de cálculo de costes se hizo evidente y necesaria. Desde entonces, han surgido aportes notables que dan cuenta del sustrato de esta línea conceptual, hasta la actualidad. Sin embargo, no existe evidencia en la literatura nacional e internacional, estudios teóricos que presenten una separación 
objetiva entre Métodos de Costeo y Herramientas de Gestión. La constatación de la falta de estudios que consideren las Herramientas de Gestión de Costos como elementos complementarios a los Métodos de Costeo busca dar respuesta a las preguntas: ¿Cómo se tratan los Métodos de Costeo y las Herramientas de Gestión en los estudios? ¿Cuál es la tendencia metodológica utilizada en los estudios que abordan el tema costos? ¿Con qué frecuencia se utilizan en los estudios los métodos de cálculo de costes y las herramientas de gestión? El artículo hace una separación clásica de los conceptos teóricos de métodos de cálculo de costes y herramientas de gestión de costes. Las Herramientas de Gestión de Costos son diferentes a los Métodos de Costeo y, cuando son elegidas por el investigador, pueden ser utilizadas de manera complementaria a los Métodos de Costeo, suplantando el rigor de los análisis; y brindar un mayor grado de seguridad en el cálculo de costos, con miras a la toma de decisiones.

Palabras clave: Sistemas de costos; Técnicas de costeo; Control de gastos.

\section{Introdução}

A contabilidade de custos, como um dos ramos da ciência contábil, houve a necessidade de se apurar e controlar os gastos com o processo produtivo. Ela se despontou após a revolução industrial e consequentemente com a ampliação e complexidade da geração de informações, decorrente dos novos processos manufatureiros no século XVIII (Fleischman \& Tyson, 1993), pois naquela época os relatórios da contabilidade tradicional não eram capazes de gerar informações adequadas para os gestores (Vanderbeck, 2015).

Esses importantes avanços contábeis estiveram intimamente atrelados tanto aos avanços da sociedade quanto das companhias, principalmente entre as últimas décadas do século XIX e as duas primeiras do século XX, onde a discussão e avanço das técnicas de apuração de custos se tornariam mais evidentes e necessárias (Beuren, 1993).

Entretanto, as contribuições de outras áreas do conhecimento, segmentadas das Ciências Sociais Aplicadas, novas escolas surgiram no início do século XX, como a Administração Científica de Taylor; as Ciências Econômicas e Políticas pelas produções de Weber; e das reflexões da nova sociedade capitalista moderna que se desenvolviam no mundo, a contabilidade também se dedicou compreender os novos fenômenos decorrentes desses avanços industriais (Sá, 2005).

Dessa forma, a escola racional de contabilidade, fundada anteriormente nos Estados Unidos, ainda no Século XIX, surgiu para mensurar com maior pragmatismo científico e exatidão qualitativa e quantitativa, os fatos e recursos operacionalizados pelas entidades; tendo, a partir de 1920 uma maior preocupação a sua missão precípua de fornecer informações relevantes à tomada de decisão e aos demais stakholders (Marion, 2018). Mas somente a partir da escola patrimonialista italiana de contabilidade de Vincenzo Masi, a contabilidade se tornou uma ciência independente (Sá, 2005).

Dessa forma, ao longo do século XX generosas contribuições se deram no desenvolvimento de controles contábeis e de apuração de custos, sob diversas óticas. Conceitos como a diferenciação de despesas e de custos; diretos e indiretos; fixos e variáveis; emergiram e ainda hoje respondem pelo substrato dessa linha conceitual, utilizados por diferentes áreas do conhecimento, além da própria contabilidade (Schmidt, 2006). No entanto, o levantamento realizado em periódicos de alto fator de impacto para os últimos cinco anos não foi constatado, tanto na literatura nacional como internacional, estudos teóricos que apresentassem uma separação objetiva entre os métodos de custeio e as ferramentas de gerenciamento de custos.

Nesse sentido, o levantamento revelou que a maioria das pesquisas utiliza como ferramenta metodológica os Estudos de Caso. Nesses estudos, o objetivo principal é adotar a escolha e aplicação de um, ou mais métodos de custeio, como forma de apresentar a melhor forma de apuração, considerando os dados de uma determinada entidade escolhida ou, segmento econômico. Entretanto, referidos estudos não consideram as "ferramentas de gerenciamento de custos" como elementos distintos e complementares aos denominados "métodos de custeios"; ao contrário, alguns autores consideram métodos e ferramentas sinônimos.

Dessa forma, este artigo objetiva provocar um novo passo em direção à discussão teórica e consensual, com vistas a fortalecer a divisão conceitual entre os Métodos de Custeio e as Ferramentas de Gerenciamento de Custos, de forma a complementar (Ramalho, 2016). Parte-se da afirmativa orientadora que as Ferramentas de Gerenciamento de Custos diferem dos 
Métodos Clássicos de Custeio utilizáveis e que, portanto, possam complementá-los inferindo responder às seguintes questões: Como os Métodos de Custeio e as Ferramentas de Gerenciamento de Custos são tratados nos estudos? Qual a tendência metodológica utilizada nos estudos que abordam a temática de custos como elemento central? Qual a frequência com que os Métodos de Custeio e as Ferramentas de Gerenciamento de Custos são utilizados nos estudos?

Como resultado, o estudo pretende contribuir à epistemologia, ao considerar uma proposta conceitual que considere aceitável que o uso complementar desses constructos, possa ampliar o grau de segurança das apurações de custos com vistas à tomada de decisão, podendo ser testada em futuras pesquisas.

\section{Referencial Teórico}

\subsection{Métodos de Custeio}

O custeio por absorção ou custeio integral, também denominado Total Absorption Costing (TAC) ou ainda Full Absorption Costing (FAC) é uma metodologia utilizada com o objetivo de avaliação e mensuração dos estoques para gerar relatórios contábeis-financeiros (Horngren et al., 2011). Esse método retrata essencialmente os custos relacionados ao processo produtivo, sendo eles diretamente alocados aos produtos ou serviços, ou seja, os custos diretos; ou alocados através de rateios, ou seja, pelos custos indiretos. Exclui ainda, os gastos administrativos e comerciais que não compõe, portanto, o custo unitário do produto. O custeio por absorção é um método que realiza a apropriação de todos os custos de produção, sejam eles fixos ou variáreis, diretos ou indiretos, aos produtos ou serviços produzidos. Uma das pessoas mais influentes durante o século XIX falando para a adoção do custeio por absorção, foi o engenheiro Alexander Hamilton Church, que desenvolveu o método da hora-máquina de alocação e aplicação de custos fixos, como energia, terra e custos de construção (Fleischman \& Tyson, 1998).

Já o custeio pleno ou método das seções homogêneas, também denominado Reichskuratorium Fur Wirtschaftlichtkeit (RKW), é um método geralmente confundido com o custeio por absorção. Enquanto o RKW aloca todos os gastos (custos e despesas) fixos e variáveis, o custeio por absorção aloca apenas os custos fixos e variáreis aos bens e serviços (Vartanian \& Nascimento, 1999). É de origem alemã, datada de 11 de novembro de 1937, quando o governo colocou em vigor o Plano de Contas Uniforme (Goering Plan) que identificava o propósito contábil de fixação de preço de venda e valoração unitária (Fleischman, 2014). Como nesse sistema custos e despesas são apropriadas, a empresa apura o total gasto na produção e venda dos produtos, considerando os gastos antes, durante e depois da venda. Portanto, os custos e despesas são primeiramente atribuídos aos setores ou departamentos de produção e posteriormente alocados aos produtos. Dessa forma, este método serve como base para fixar o preço de venda do produto, uma vez que, calculados os custos e as despesas, bastando acrescentar a margem de lucro que a empresa almeja.

Outro método é o custeio direto, por vezes denominado custeio variável ou ainda custeio marginal. Na literatura internacional é conhecido como Direct Costing ou Variable Costing ou ainda como Marginal Costing. A origem metodizada desse método se deu por meio da publicação do artigo “What did we earn last month?” de Jonathan Harris em 1936 (Frost, 1968). Trata-se de um método em que somente os custos e despesas variáveis são alocados aos produtos ou serviços, uma vez que os custos e despesas fixas são consideradas despesas do período, e portanto, seus valores são alocados diretamente ao resultado do período (Horngren et al., 2011). É assim chamado por que os gastos variáveis, na sua maioria, são diretamente alocados. Tem em vista que os normativos de tributação sobre a renda no Brasil determinam o uso do custeio por absorção, como se observa, por exemplo, no art. 13 do Decreto-Lei n 1.598/1977. Assim, o custeio variável, assim como outros métodos de custeio são utilizados para fins gerenciais, ou seja, como ferramenta de auxílio a administração para a tomada de decisões.

Outra forma de custeamento é o custeio baseado em atividades, também conhecido como Activity-Based Costing (ABC). Trata-se de um método desenvolvido na renomada universidade de Harvard em meados da década de 1980 com o objetivo de levantar os custos das diferentes atividades da empresa e entender seu comportamento, encontrando direcionadores que 
representem as relações entre os produtos e as atividades (Cooper \& Kaplan, 1987). O ABC foi desenvolvido para corrigir graves deficiências dos sistemas tradicionais, que, de modo geral, utilizavam apenas três categorias de gasto: mão-de-obra, matériaprima e gastos gerais. Desse modo, as companhias de manufaturas consideram a mão-de-obra e a matéria-prima por unidade, entretanto, os custos indiretos eram denominados custos gerais (Kaplan \& Anderson, 2007). O ABC pretende deixar o cálculo dos custos dos produtos menos subjetivo, pois deixa de alocar os custos indiretos fixos aos produtos por critérios de rateio arbitrários que distorcem os custos dos produtos (Cooper \& Kaplan, 1998). Assim, esse método se utiliza de direcionadores, que podem ser classificados em três tipos: de transação, de duração e de intensidade. Assim, o ABC se concentra nos custos indiretos, superando as deficiências dos métodos de alocação de custos tradicionais que aproximam as informações da produção com a contabilidade financeira (Horngren et al., 2011). Esse método é indicado para as preferencialmente para as atividades-meio das entidades, uma vez que permite visualizar o impacto delas nos preços dos produtos e serviços (Bornia, 2010).

Um variação ao $\mathrm{ABC}$ tradicional, c custeio baseado em atividades e tempo, também conhecido como Time-Driven Activity-Based Costing (TDABC). Esse método foi desenvolvido por Kaplan e Anderson em 2004 para ajustar e aprimorar o método de custeio ABC tradicional. Nas pesquisas efetuadas por Kaplan e Anderson (2007) sobre a implantação do ABC, constataram-se problemas, como alto custo, tempo e dificuldades na validação dos dados. O TDABC além de simplificar o processo de alocação de custos, também elimina a necessidade de se entrevistar os colaboradores e de se pesquisar a alocação dos custos dos recursos às atividades antes de alocá-los para os três principais objetos de custos, ou seja, os pedidos, os produtos e os clientes. O novo método atribui os custos dos recursos diretamente aos objetos de custo, utilizando uma estrutura simplificada e, que exige apenas dois conjuntos de estimativas (Kaplan \& Anderson, 2007). Assim, o TDABC parte da premissa de se alocar os custos indiretos por unidade de tempo de capacidade e o tempo necessários para a realização de uma atividade, ou seja, apresenta de maneira mais coerente os custos aos produtos.

Outro método de custeamento é a Contabilidade de Consumo de Recursos (CCR) ou ainda Resource Consumption Accounting (RCA). Esse método é uma variação do método alemão de custeio denominado Grenz Plan Kostenrechnung (GPK) com o método de custeio ABC para formar um sistema contábil abrangente para controle gerencial e que pode finalmente fornecer informações não compostas para tomar decisões (Mackie, 2006). A RCA integra medição e gerenciamento do negócio em um sistema de contabilidade que reflete a forma como a empresa realmente opera. Para tanto, existem três pontos para se compreender o RCA: a visão dos recursos, o modelo baseado em quantidade e a natureza dos custos (Polejewski, 2007).

Primeiro ponto, o método se concentra nos custos operacionais e no consumo de recursos, e os centros de custo escolhidos refletem isso. O modelo utiliza mais centros de custo do que métodos tradicionais de contabilidade. Os recursos de cada centro de custo devem ser homogêneos e devem ser da responsabilidade de apenas um gerente. Ao agrupar recursos em torno de uma medida de saída simplificada, os centros de custo são mais fáceis de gerenciar. Segundo ponto, é que ele se contrapõem o método do custeio tradicional para explicar relacionamentos, pois é baseado em valores monetários. Entretanto, expressar essa relação em dinheiro exige distorções de custos fixos. Assim, ao usar relacionamentos quantitativos baseados na causalidade, o RCA produz resultados mais precisos como modelo preditivo. Já o terceiro e último ponto, diz respeito a separação dos custos de acordo com seus comportamentos, enquanto o custeio tradicional assume que todos os custos são variáveis.

Sua origem se deu no início do século XXI com o objetivo auxiliar a gestão financeira e contábil das entidades. Durante quase uma década seus idealizadores ficaram amadurecendo os seus princípios e em 2008 foi fundado o Resource Consumption Accounting Institute, com o intuito de tornar essa prática mais conhecida e mais comum no mercado de trabalho (RCA Institute, 2008). Em 2009 o IFAC - Committe International Federation of Accountants realizou uma publicação onde o RCA passou a ser formalmente reconhecido como um método de custeio (IFAC, 2009). 


\subsection{Ferramentas de Gerenciamento de Custos}

A primeira e mais utilizadas das ferramentas para fins gerenciais é a análise do custo-volume-lucro ou Cost-VolumeProfit Analysis (CVP) é uma ferramenta de gerenciamento relacionada ao custeio variável, pois é a partir dele que se calcula cinco importantes indicadores: a margem de contribuição, o ponto de equilíbrio (contábil, financeiro e econômico), a margem de segurança e a alavancagem operacional (Mowen et al., 2017). Resumidamente, a margem de contribuição ou contribution margin ratio (CMR) é a diferença entre o preço de venda unitário e os custos variáveis unitários, ou ainda, quantia disponível para cobrir os custos fixos e gerar lucro. Já o ponto de equilíbrio, também denominado de ponto crítico, ou ponto de ruptura ou ainda como break-even point (BEP), por sua vez, é o ponto em que a margem de contribuição se iguala aos custos e despesas fixos, ou seja, é o ponto em que a organização não apresenta nem ganhos, nem perdas. (Bornia, 2010). A análise pela margem de contribuição é denominada na Alemanha de custeio Grenz Plan Kostenrechnung (GPK). O GPK é um dos métodos de custeio mais utilizados nas companhias alemãs, fornecendo o suporte ideal para tomada de decisão de curto prazo, considerando-se o fato de não adicionar ao custo do produto o custo fixo da entidade (Friedl et al., 2005). Já a margem de segurança é o intervalo existente entre o volume de vendas e o BEP, ou seja, ela permite que uma empresa saiba qual é o valor exato que ganhou ou perdeu e se eles estão acima ou abaixo do ponto de equilíbrio (Mowen et al., 2017). Por fim, a alavancagem operacional ou operational leverage, que estuda a combinação relativa aos custos fixos e custos variáveis. Uma vez negociados, os custos fixos podem ser convertidos em custos variáveis (Mowen et al., 2017). Caso os custos fixos forem usados para reduzir os custos variáveis, de modo que a margem de contribuição aumente e a receita operacional diminua, o nível de alavancagem operacional aumenta, o que sinaliza um aumento de risco do negócio. A análise do custo-volume-lucro deve ser utilizada para uma análise marginal de curto prazo, pois assume que os custos variáveis unitários e as receitas unitárias são constantes, o que é adequado para pequenas variações na produção e nas vendas.

Outra ferramenta de gerenciamento de custos é o custeio-alvo, ou custeio-meta, ou ainda target costing é uma ferramenta de gerenciamento de custos é de origem japonesa do final da década de 1960, entretanto é atribuído ainda, à companhia norteamericana General Eletric (GE), durante a segunda guerra mundial (1939-1945) (Burrows \& Chenhall, 2012). É tratada como uma técnica de engenharia de valor, ou seja, reduzir custos e prevenir eventuais custos desnecessários antes de produzir o produto ou serviço (Csillag, 2009). Assim, ela tem como objetivo eliminar os gastos que não agregam valor ao produto ou serviço. O custeio-alvo é um sistema de idealização de lucros e gerenciamento de custos, orientado pelo preço de venda, ou seja, focado no cliente (Ansari et al., 2010). Uma das vantagens desse recurso está no planejamento dos gastos por meio da visão de mercado e redução de custos do produto, considerando as necessidades do cliente e o preço praticado pela concorrência. Dessa forma, a opinião do cliente é um parâmetro para o desenvolvimento do processo de produção, para incorporar decisões de qualidade, custo e tempo na análise do custo de produção. Trata-se, portanto, do custo do processo produtivo que se precisa atingir para garantia da obtenção de um preço competitivo no mercado e que, ao mesmo tempo, propicie ganhos justos à organização (Colauto et al., 2004).

O custeio Kaizen, por sua vez, é uma ferramenta de gerenciamento de custos utilizada no processo produtivo para a inovação e sua manutenção, ou seja, manter os modelos atualizados sobre tecnologia, negócios e operações (Sakurai, 1997). É semelhante ao custeio-alvo, pois também trabalha sistematicamente para reduzir os custos a valores desejados. Entretanto, não se preocupa apenas com o sistema financeiro, mas também com a produção e com a qualidade dos produtos. O custeio-alvo, por exemplo, tem como objetivo apoiar o processo de redução de custos na fase de desenvolvimento dos novos modelos. O custeio Kaizen, por sua vez, tem como objetivo a apoiar o processo de redução de custos dentro do contexto do atual sistema produtivo e dos atuais produtos fabricados.

A diferenciação entre as ferramentas se faz necessária, pois, enquanto o custeio Kaizen busca um conjunto amplo de pequenas melhorias na produção atualmente realizada, a lógica do custeio-alvo pode implicar a necessidade da introdução de 
inovações tecnológicas radicais na fase de desenvolvimento de produto e dos processos de fabricação (Monden, 1999). Esse método se preocupa ainda com a redução de custos para cada produto e competência, necessitando de produtos e peças padronizadas e da aplicação de engenharia de valor, para aumentar a eficiência do uso do equipamento e a eficácia dos custos indiretos, priorizando os gargalos no fluxo de trabalho, tendo como resultado a redução de custos e de materiais diretos e de mão-de-obra direta. Assim, também guarda certa relação com a teoria das restrições ou theory of constraints (TOC) de Eliyahu M. Goldratt.

Assim, tem-se mais uma variação como ferramenta de gerenciamento de custos denominada Contabilidade de Ganhos ou Throughput Accounting. Trata-se de uma ferramenta de gerenciamento de custos que fornece aos gestores informações de suporte à decisão para a melhoria da lucratividade da organização, pois se apresenta como uma medida de desempenho da Teoria das Restrições (Corbett, 1998). Essa técnica se utiliza de três medidores de sucesso. Assim, se afirma que qualquer coisa pode ser classificada em três medidas: ganho; investimento; e despesa operacional. O ganho (throughput) ou margem variável pura, é a taxa na qual o sistema gera dinheiro por meio das vendas, sendo representado pela diferença entre a receita bruta da empresa e os custos totalmente variáveis. Já o investimento é todo dinheiro que o sistema investiu na compra de insumos. Por fim, a despesa operacional é todo o dinheiro que o empreendimento emprega para transformar o estoque em ganho (Corbett, 1998). O objetivo das companhias, segundo essa ferramenta é focar no aumento do ganho, pois das três medidas o ganho (lucro) é o único que não tem limites. Por outro lado, tanto o investimento e quanto as despesas operacionais devem ser diminuídos em direção ao limite possível, no caso, zero.

Outra ferramenta de gerenciamento de custos é a denominada Contabilidade Enxuta ou Lean Accounting é uma ferramenta de gerenciamento de custos que apoia a manufatura enxuta, também denominada de Lean Manufacturing e, que fora desenvolvida pela indústria de veículos japonesa Toyota entre os anos de 1948 e 1975 (Ohno, 1988). Existem dois princípios básicos na Contabilidade Enxuta. O primeiro é a utilização de métodos simples aos processos financeiros, de controle e de medição da organização. Isso não é diferente de aplicar métodos simples a qualquer outro processo. O objetivo é eliminar o desperdício, liberar a capacidade, agilizar processos, e eliminar erros e defeitos. Já o segundo princípio é a mudança fundamentalmente aos processos de contabilidade, controle e medição, de modo que eles motivem a mudança enxuta e melhorias ao fornecimento de: informações apropriadas para controle e tomada de decisões; à compreensão do valor do consumidor; à avaliação correta sobre o impacto financeiro da melhoria magra; sendo, eles mesmos, simples, visuais e de baixos resíduos (Maskell et al., 2011).

Por outro lado, o Custeio Padrão ou Traditional Standard Costing (TSC) ou por vezes denominado Standard Cost Accounting (SCA) é uma ferramenta utilizada no suporte ao controle e acompanhamento da produção na indústria. Dessa forma, a medição de custos fica em segundo plano (Horngren et al., 2011). Esse método tem como objetivo simular uma base comparativa entre os custos reais incorridos com os custos que deveriam ser incorridos. Por meio da comparação entre o padrão e o real é analisada a fim de levantar as causas que levaram aos desvios (Silva et al., 2011). O cálculo dos custos reais é realizado pela fixação de padrões de consumo por um padrão monetário. A utilização desse método é eficiente para o controle de matériasprimas consumidas no produto, pois é sensível a sua variação, mas é ineficiente no controle dos custos indiretos, pois tem dificuldades de estipular um padrão de comportamento para esses custos (Iudícibus et al., 2003). Deve ser analisado dessa forma se os gastos incorridos no período foram bem ou mal aplicados pelas áreas de apoio. Sua origem é da década de 1920 é um método central na contabilidade gerencial praticada hoje porque é usado para relatórios de demonstração financeira para avaliação de demonstração de resultados e itens de linha do balanço, como custo das mercadorias vendidas (CMV) e avaliação de inventário (Chatfield \& Vangermeersch, 1996).

Já o Custeio Baseado em Processos ou Process-Based Costing (PBC) é uma técnica de controle de custos que se baseia na análise dos processos encobertos às atividades, por meio de fluxogramas, da entidade desde a compra de insumos, a sua 
transformação até a venda ou prestação de serviços (Lee et al., 2003) (Mocciaro et al, 2012). Reúne informações que suportam a otimização da cadeia de valor das empresas e melhora o sistema de controle do custeio baseado em atividade, ao informar os gerentes sobre controle da qualidade, tempo de produção e, principalmente, satisfação do cliente. Portanto, é útil para medir os resultados de custos dos esforços na melhoria da qualidade (Nadeau et al., 2010). Trata-se de uma variação do custeio baseado em atividades. As primeiras obras são do início dos anos de 1990 (Lawson, 1994) (Sievanen \& Tornberg, 2002).

Por fim, temos ainda a ferramenta denominada Unidade de Esforço de Produção (UEP) ou Production Effort Unit (PEU) é um método de custeio fundamentado no esforço que a organização despende para transformar matéria-prima em produto final (Bornia, 2010). Este método foi desenvolvido por George Perrin, também denominado método GP, no final da década de 1930. Anteriormente, era denominado método Unidades de Produção ou Unitéde Production (UP), posteriormente de método Unidades de Valor Adicionado ou Unité de Valeur Ajouté (UVA) ou, para romper com referências anteriores à noção de produção (Levant \& De la Villarmois, 2001). A principal vantagem do método GP e os desenvolvimentos posteriores nele, foi a simplificação dos custos. Isto é baseado no princípio dos "custos ocultos". Atualmente, o principal argumento dos defensores desse método está na possibilidade de calcular, por meios realistas, o retorno de cada uma das contas da empresa (Fievez et al, 1999). Esse método simplifica o cálculo da produção por meio da fixação de uma unidade de medida comum a todos os produtos e processos da fábrica. Essa unidade representa o trabalho realizado para a execução ou ainda necessário para a produção em cada posto operativo (PO). Assim, para o funcionamento do método é que cada PO realiza um esforço de produção, conhecido como potencial produtivo. Esse potencial é a capacidade produtiva por unidade de tempo (UEP/h) (Bornia, 2010).

\section{Metodologia}

A pesquisa bibliográfica é desenvolvida com base, principalmente, de livros e artigos científicos, sendo que a principal vantagem desse tipo de pesquisa reside no fato de permitir ao investigador detectar dados que estão dispersos no tempo e espaço, para construir o problema de pesquisa e o objetivo de estudo (Gil, 2018). Essas concepções também são consideradas premissas que emergem da análise da literatura, como forma de identificar o problema de pesquisa e objetivo de estudo (Yin, 2015).

Desta forma, a condução operacional deste estudo partiu da análise quantitativa distinguindo preferências, comportamentos e outras ações que, em conformidade ao método utilizado, constatou a falta de pesquisas que buscassem tratar as Ferramentas de Gestão de Custos de maneira complementar aos Métodos de Custeio (Estrela, 2018). O processo de escolha e análise dos dados foi operacionalizado, devendo, pela análise sistemática da literatura, contribuir pela sintetização dos conceitos clássicos dos constructos, neste caso, sobre os métodos de custeio e as ferramentas de gerenciamento de custos.

O levantamento bibliográfico foi realizado nos portais PROQUEST, SCIELO e SPELL em periódicos de alto fator de impacto, superior a A2 segundo a classificação da CAPES, compreendendo artigos nacionais e internacionais. Os filtros foram direcionados a eleger as publicações dos últimos cinco anos, identificando no Título, no Resumo e nas Palavras-Chave, os termos: Contabilidade de Custos; ou Métodos de Custeio; ou Ferramentas de Gerenciamento de Custos, retornando 335 artigos para os três portais.

Sobre os resultados preliminares, filtrou-se no Assunto, o tema Contabilidade, sendo aplicados filtros que excluíssem da base de dados, artigos que tratassem do tema Custos Governamentais; Visão Baseada em Recursos (RBV) de Barney; e de Planejamento Estratégico, como os direcionadores de custos de Porter, para não enviesar o levantamento. Sob os novos filtros os resultados retornaram para os portais PROQUEST, SCIELO e SPELL, respectivamente, 42, 14 e 35 publicações, sendo escolhidos a partir da leitura dos resumos e, pela convergência ao estudo, 36, 11 e 33 artigos, respectivamente. 


\section{Resultados e Discussão}

Como verificado na Introdução, este estudo decorre da seguinte afirmativa orientadora: que as Ferramentas de Gerenciamento de Custos diferem dos Métodos Clássicos de Custeio, e que, portanto, possam transitar de maneira complementar, face às metodologias de custeio elegíveis pelos pesquisadores. A principal justificativa para uso da afirmação orientadora foi o estudo de Ramalho, (2016), que considerou analisar as características dos Sistemas de Custos entre entidades do setor automobilístico e de máquinas e equipamentos, alemães, instalados no Brasil, compreendendo os Métodos de Custeio e as Ferramentas de Gestão utilizadas pelas empresas.

O levantamento bibliográfico constatou, inicialmente, que nenhum dos artigos pesquisados esclarecia sobre a separação conceitual entre os métodos de custeio e as ferramentas de gestão. A pesquisa revelou ainda, que $89 \%$ dos estudos que tratam sobre métodos de custeio referem-se a Estudos de Caso, sendo que 67 dos 80 artigos selecionados tendem ao principal objetivo de eleger um método de custeio, para tratar os dados da entidade escolhida, com 61 artigos; ou utilizaram mais de um método para comparar a melhor forma de aplicação a um determinado segmento econômico, totalizando 6 artigos. Os 13 artigos remanescentes também são Estudos de Caso e discutem especificamente, custos logísticos. Essas constatações fomentaram a produção deste estudo, e permitiram apresentar a Tabela 1 a seguir, que reflete esta tendência:

Tabela 1: Frequência de artigos sobre métodos de Custeio e de Ferramentas de Gerenciamento de Custos.

\begin{tabular}{l|c|c|c|c|c|c}
\hline \multicolumn{1}{c|}{ Bases } & \multicolumn{2}{|c|}{ PROQUEST } & \multicolumn{2}{c}{ SCIELO } & \multicolumn{2}{c}{ SPELL } \\
\hline Metodologia & $\begin{array}{c}\text { Estudos de } \\
\text { Caso }\end{array}$ & $\begin{array}{c}\text { Outros } \\
\text { Estudos }\end{array}$ & $\begin{array}{c}\text { Estudos } \\
\text { de Caso }\end{array}$ & $\begin{array}{c}\text { Outros } \\
\text { Estudos }\end{array}$ & $\begin{array}{c}\text { Estudos } \\
\text { de Caso }\end{array}$ & $\begin{array}{c}\text { Outros } \\
\text { Estudos }\end{array}$ \\
\hline Métodos de Custeio & 31 & 5 & 11 & 0 & 29 & 4 \\
\hline $\begin{array}{l}\text { Ferramentas de Gerenciamento de } \\
\text { Custos }\end{array}$ & 2 & 0 & 0 & 0 & 1 & 0 \\
\hline
\end{tabular}

Fonte: Autores.

A Tabela 1 acima revela uma variação aparentemente maior de artigos, se comparada ao total de artigos analisados nos portais (36 PROQUEST; 11 SCIELO; e 33 SPELL), pois correspondem ao fato dos autores considerarem as Ferramentas de Gerenciamento de Custos como termos similares aos Métodos de Custeio, sendo que, na prática, os estudos utilizaram- se apenas os métodos de custeio apontados no referencial teórico.

Conquanto, nenhum dos trabalhos pesquisados considerou as Ferramentas de Gerenciamento de Custos como elementos complementares ou de apoio aos Métodos de Custeio; tais concepções reforçaram a necessidade de separação conceitual pretendida nesse estudo. Desta forma, constatou-se que na literatura nacional e internacional, os estudos não apresentam uma separação objetiva entre os métodos de custeio e as ferramentas de gerenciamento de custos; ao contrário, conforme já mencionado, em três produções constatou-se que os temas "ferramentas" e "métodos" são tratados como sinônimos, não complementares.

O estudo também propiciou a realização de um quadro sinótico que, para fins didáticos, é apresentado com o objetivo de aclarar o fundamento complementar e de apoio das Ferramentas de Gerenciamento de Custos face aos Métodos de Custeio elegíveis nos estudos.

Na Tabela 2 nota-se que, com exceção da Ferramenta "Análise do Custo-Volume-Lucro", que se relaciona especificamente ao Método de Custeio Direto, as demais Ferramentas de Gerenciamento não têm, por princípio conceitual, relação direta ou específica com algum Método de Custeio. 
Tabela 2: Métodos de Custeio X Ferramentas de Gerenciamento de Custos.

\begin{tabular}{c|c}
\hline Métodos de custeio & Ferramentas de gerenciamento \\
\hline $\begin{array}{c}\text { 1. Custeio direto, ou custeio variável, ou ainda, } \\
\text { custeio marginal } \\
\text { (Direct Costing or Variable Costing or Marginal } \\
\text { Costing) }\end{array}$ & $\begin{array}{c}\text { 1. Análise do custo-volume-lucro } \\
\text { (Cost-Volume-Profit Analysis - CVP) }\end{array}$ \\
\hline $\begin{array}{c}\text { 2. Custeio pleno ou método das seções homogêneas } \\
\text { (Reichskuratorium Fur Wirtschaftlichtkeit - RKW) }\end{array}$ & $\begin{array}{c}\text { 2. Custeio-alvo ou custeio-meta } \\
\text { (Target Costing - TC) }\end{array}$ \\
\hline $\begin{array}{c}\text { 3. Custeio por absorção ou custeio integral } \\
\text { (Total Absorption Costing - TAC or Full Absorption } \\
\text { Costing - FAC) }\end{array}$ & 3. Custeio Kaizen \\
\hline $\begin{array}{c}\text { 4. Custeio baseado em atividades } \\
\text { Activity-Based Costing - ABC) }\end{array}$ & $\begin{array}{c}\text { 4. Contabilidade de Ganhos } \\
\text { (Through-put Accounting - TA) }\end{array}$ \\
\hline $\begin{array}{c}\text { 5. Custeio Baseado em Atividades e Tempo } \\
\text { (Time-Driven Activity-Based Costing - TDABC) }\end{array}$ & $\begin{array}{c}\text { 5. Contabilidade Enxuta } \\
\text { (Lean Accounting - LA) }\end{array}$ \\
\hline $\begin{array}{c}\text { 6. Contabilidade de Consumo de Recursos } \\
\text { Resource Consumption Accounting - RCA) }\end{array}$ & $\begin{array}{c}\text { 6. Unidade de Esforço de Produção - UEP } \\
\text { (Production Effort Unit- PEU) }\end{array}$ \\
\hline & $\begin{array}{c}\text { 7. Custeio Padrão } \\
\text { (Standard Cost Accounting - SCA) }\end{array}$ \\
\hline
\end{tabular}

Fonte: Autores.

Entretanto, os conceitos clássicos dos Métodos de Custeio e das Ferramentas de Gestão permitem que pesquisadores escolham ferramentas específicas, conforme suas características peculiares, por exemplo, as ferramentas de "Custeio Padrão" ou "Custeio Baseado em Processos": que podem ser escolhidas como as melhores ferramentas complementares, quando os Métodos de Custeio utilizados forem os do tipo "ABC" ou "TDABC".

Por fim, os resultados obtidos permitiram responder às questões da pesquisa, ao constatar-se que: 1. Os Métodos de Custeio e as Ferramentas de Gerenciamento de Custos são tratados nos estudos como sinônimos. 2. A tendência das pesquisas sobre os sistemas de Custos estão focadas a utilizarem a ferramenta metodológica Estudo de Caso. 3. As Ferramentas de Gerenciamento de Custos são negligenciadas nas pesquisas, e tendem não ser utilizadas de maneira complementar aos Métodos de Custeio escolhidos. Essas constatações permitiram convergir como proposição conclusiva de que as Ferramentas de Gerenciamento de Custos se distinguem dos Métodos de Custeio e, portanto, podem ser usadas de maneira complementar, suplantando o rigor das análises, quando eleitas pelo pesquisador.

\section{Considerações Finais}

As produções acadêmicas que buscam aplicar métodos teóricos de custos em Estudos de Caso não são errôneas, ao contrário trazem alento para muitas das aplicações práticas aos gestores. Entretanto, o viés de apresentar o melhor método de custeio aplicável para empresas ou segmentos setoriais, sem considerar a complementaridade das Ferramentas de Gestão, reduz a abrangência desses estudos; por não esclarecerem ao universo prático das empresas, que o uso dessas ferramentas, possa transitar pelas metodologias de custeio escolhidas, propiciando aumento na eficiência das apurações e, consequentemente à 
tomada de decisões.

Dessa forma, este artigo não pretendeu discutir a existência de eventuais falhas nas produções acadêmicas, no sentido de criticar se os modelos de apuração de custos propostos pelos autores, nos Estudos de Caso, estariam corretos; mas por considerar que essas pesquisas seriam ainda mais robustas, se também considerassem, de maneira complementar, as Ferramentas Gerenciais de Custos. O aprofundamento da análise dos dados permitiu identificar que as Ferramentas de Gerenciamento de Custos têm sido negligenciadas nos estudos, ou tratadas como sinônimos de Métodos de Custeio.

Como contribuição o estudo remonta uma classificação teórica dos Métodos de Custeio em separado das Ferramentas de Gerenciamento de Custos, para propor uma divisão conceitual factível, para que futuras pesquisas considerem utilizar, como metodologia, os dois constructos de maneira complementar, elevando a eficiência tanto das abordagens empíricas quanto teóricoconceituais.

\section{Referências}

Brasil. Decreto-lei n. 1.598, de 26 de dezembro de 1977. Altera a legislação do imposto de renda. http://www.planalto.gov.br/ccivil_03/decretolei/del1598compilado.htm.

Ansari, S. L. et al. (2010). Target costing. LaVergne, Mountain Valley Publishing.

Beuren, I. M. (1993). Evolução histórica da contabilidade de custos. Contabilidade Vista \& Revista, 5, (1), 61-66.

Bornia, A. C. (2010). Análise gerencial de custos: aplicação em empresas modernas. Atlas.

Burrows, G. \& Chenhall, R. H. (2012). Target costing: first and second comings. Accounting History Review, 22 , (2), $127-142$.

Cha Tfield, M. \& V Angermeersch, R. (1996). The history of accounting: an international encyclopedia. Garland Science.

Colauto, R. D.; et al. (2004). O custeio variável e o custeio-alvo como suportes às decisões de investimentos, no desenvolvimento de novos produtos. Revista de Administração e Contabilidade da Unisinos, 1, (2), 33-41.

Cooper, R. \& Kaplan, R. S. (1987). Chapter 8: How cost accounting systematically distorts product cost. In: Accounting \& management: field study perspectives. Harvard Business Press.

Cooper, R. \& Kaplan, R. S. (1998). Design of cost management systems. Pearson.

Corbett, T. (1998). Throughput accounting. Great Barrington, North River Press.

Csillag, J. M. (2009). Análise do Valor. Atlas.

Estrela, C. (2018). Metodologia científica: ciência, ensino e pesquisa. Artes Médicas.

Fievez, J. et al. (1999). La methode UVA: Du contrôle de gestion à la maîtrise du profit: une approche nouvelle en gestion. Dunod.

Fleischman, R. K. (2014). Chapter 4: A history of management accounting through the 1960s. In: Accounting history from therenaissanceto the present: a remembrance of Luca Pacioli. Routledge, 119-142.

Fleischman, R. K. \& Tyson, T. N. (1993). Cost accounting during the industrial revolution: the present state of historical knowledge. The Economic History Review, 46, (3), 503-517.

Fleischman, R. K. \& Tyson, T. N. (1998). The evolution of standard costing in the U.K. and U.S.: from decision making to control. Abacus, 34, (1), 92-119.

Friedl, G. et al. (2005). Relevance added: combining ABC with German cost accounting. Strategic Finance, 86, (12), 56-61.

Frost, E. L. (1968). Direct costing for external financial reporting. Thesis in Accounting. Master of Business Administration-Lubbock: Texas Tech University. Horngren, C. T. et al. (2011). Cost accounting: a managerial emphasis. Prentice Hall.

Gil, A. C. (2018). Como elaborar projetos de pesquisa. Atlas.

IFAC. (2009) Evaluating and improving costing in organizations. International Good Practice Guidance, n. Professional Accountants in Business Committee. International Federation of Accountants.

Iudícibus, S. et al. (2003). Dicionário de termos de contabilidade. Atlas.

Kaplan, R. S. \& Anderson, S. R. (2007). Time-Driven Activity-Based Costing: A Simpler and More Powerful Path to Higher Profits. Harvard Business School Press.

Lawson, R. A. (1994). Beyond ABC: process-based costing. Journal of Cost Management, 8, (3), 33-44. 
Research, Society and Development, v. 10, n. 9, e59710918638, 2021

(CC BY 4.0) | ISSN 2525-3409 | DOI: http://dx.doi.org/10.33448/rsd-v10i9.18638

Lee, R. H. et al. (2003). Process-based costing. Journal of Nursing Care Quality, 18, (4), 259- 266.

Levant, Y. \& De la Villarmois, O. (2001). La methode GP: apports et applications d'une methode alternative de calcul des couts. 22 Ème Congres de L'AFC. Anais... In: CONGRES DE L’AFC. France. https://halshs.archives-ouvertes.fr/halshs-00584658.

Mackie, B. (2006). Merging GPK and ABC on the Road to RCA: Toronto's hospital for sick children has successfully implemented the first part of a new accounting system that provides relevant information for its operations managers to use on the job. Strategic Finance, 88, (5), 33-40.

Martin, J. R. (2017). Chapter 11: Conventional Linear Cost-Volume-Profit Analysis. Management accounting: concepts, techniques \& controversial issues Management and Accounting Web.

Marion, J. C. (2018). Contabilidade empresarial: instrumentos de análise gerência e decisão. Atlas.

Maskell, B. H. et al. (2011). Practical lean accounting: a proven system for measuring and managing the lean enterprise. Productivity Press.

Mocciaro li Destri, A. et al. (2012). Bringing strategy back into financial systems of performance measurement: Integrating EVA and PBC. Business Systems Review, 1, (1), 85-102.

Monden, Y. (1999). Sistema de redução de custos: custo-alvo e custo Kaizen. Bookman.

Mowen, M. M. et al. (2017). Managerial accounting: the cornerstone of business decision-making. South-Western College Pub.

Nadeau, M.-C. et al. (2010). A dynamic process-based cost modeling approach to understand learning effects in manufacturing. International Journal of Production Economics, Integrating the Global Supply Chain. 128, (1), 223-234.

Ohno, T. (1988). Toyota production system: beyond large-scale production. Productivity Press.

Polejewski, S. A. (2007). Resource Consumption Accounting. University of St. Thomas.

Ramalho, L. D. F. (2016). Estudo sobre sistemas de custos gerenciais de subsidiárias alemãs em operação no Brasil: um enfoque da nova teoria institucional. Dissertação de Mestrado-São Paulo: Universidade de São Paulo.

RCA Institute. (2008). What is RCA? http://www.rcainstitute.org/rcai-3-WhatIsRCA.php.

Sá, A. L. (2005). Doutrinas, escolas novas razões de entendimentos na Ciência Contábil. Revista Catarinense da Ciência Contábil, 4, (10), 25-34.

Sakurai, M. (1997) Gerenciamento integrado de custos. Atlas.

Sievanen, M. \& Tornberg, K. (2002). Process-based costing: The best of activity-based costing. AACE International Transactions, 15.1-15.6.

Silva, L. I. S. et al. (2011). O custeio padrão como instrumento de controle e informação gerencial: uma proposta de implementação do custo padrão x real numa cooperativa de laticínios. Anais do Congresso Brasileiro de Custos - ABC. Anais... In: XVIII CONGRESSO BASILEIRO DE CUSTOS. Associação Brasileira de Custos, https://anaiscbc.emnuvens.com.br/anais/article/view/582.

Schmidt, P. (2006). História do pensamento contábil. Atlas.

Vanderbeck, E. J. (2015). Principles of cost accounting. Cengage Learning.

Vartanian, G. H. \& Nascimento, D. T. (1999) O método de custeio pleno: uma abordagem conceitual. Anais do Congresso Brasileiro de Custos - ABC. Anais... In: VI Congresso Brasileiro De Custos. Associação Brasileira de Custos, https://anaiscbc.emnuvens.com.br/anais/article/view/3170.

Yin, R. K. (2015). Estudo de caso: planejamento e métodos. Bookman. 